\title{
Search Complexities for HTN Planning
}

\author{
Ronald Alford ${ }^{1}$
}

Published online: 22 September 2015

(C) Springer-Verlag Berlin Heidelberg 2015

\section{Introduction}

From home assistance robots [7] tointeractive instructions and diagnostics [6], Hierarchical Task Network (HTN) planning is key formalism for systems that intelligently respond to human interaction. HTNs represent procedural knowledge on how to break down complex tasks into executable steps, and have also been used in planning for robotics [14], security testing [13], and plan recognition [5]. In large part, HTN planning extends classical planning [10], which focuses on using domain-independent heuristics to search for a sequence of actions to obtain a goal. Over the past 15 years, there have been significant advances in heuristics and heuristic search algorithms for classical planning [11, 12, 15], but for the most part, these techniques have not been incorporated into modern HTN planners.

This thesis [1] lays out the theoretical foundations necessary to adapt domain-independent heuristic search to HTN planning. To that end, this thesis discusses three areas: decidable subsets of HTN planning and their decision procedures, the theoretical difficulty of directly adapting domain-independent heuristics, and a translation of certain HTN problems directly into classical planning, so that any classical planner can be used for heuristic HTN search.

Ronald Alford

ronwalf@volus.net

14555 Overlook Avenue SW, Code 5514, 20375 Washington, DC, USA

\section{Results}

Although HTN planning is in general undecidable [8], this thesis identifies a number of widely-met restrictions on problem syntax that restore decidability, as well as algorithms that solve these problems. Analyzing decidable fragments of HTN planning allows for theoretical guarantees on planner performance, and more practically useful, the decision procedures for these fragments can identify unsolvable problems, which is required by a number of planning applications [4]. Later work has extendedthe results of this thesis to provide tight complexity bounds for a wide variety of syntactically constrained HTN problems $[2,3]$.

Even though the decision procedures provide termination guarantees, their worst case performance is still highly impractical, and so HTN planners may need heuristics to effectively search for solutions. Many of the successful domain-independent heuristics for classical planning rely on a technique called delete relaxation [12]. Delete relaxation makes a problem easy to solve by transforming the problem's actions so that actions can only add information to the problem's state. One can trivially solve delete-relaxed problems by greedily applying actions until a fixed point is found, and then checking whether the goal holds in the fix-point state. However, this thesis shows that deciding whether a delete-relaxed HTN planning problem has a solution is NP-complete. Barring a major breakthrough, any algorithm for solving such problems will take exponential time in the worst case.

Although efficiently computable delete-relaxation derived heuristics with full HTN semantics are out of reach (unless $\mathrm{P}=\mathrm{NP}$ ), the thesis gives two potential paths to HTN heuristic search. The first, more theoretically focused, is that an alternate set of HTN semantics, called HTN 
Fig. 1 Fast-Forward's (FF's) CPU time in an office-delivery domain, with and without the translated HTN knowledge. In the graph at left, the number of packages to deliver is fixed at 40 and the number of reachable rooms varies. In the graph at right, the number of rooms is fixed at 40 and the number of packages varies
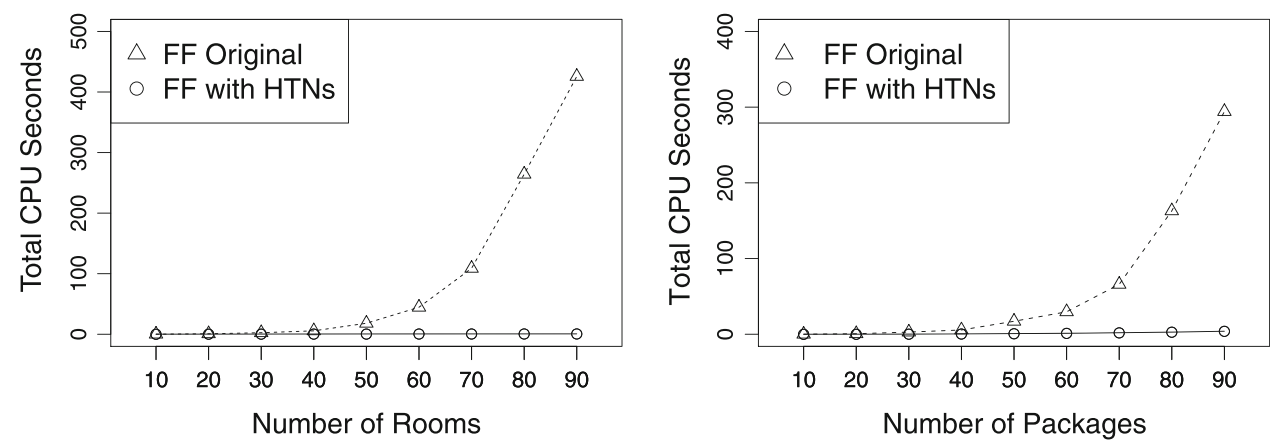

planning with Task Insertion [9], is polynomial-time decidable when delete-relaxed. Secondly, the thesis gives a translation of a common class of HTN problems into classical planning problems. Experiments from the thesis, excerpted in Fig. 1, show that a small amount of translated HTN knowledge can dramatically improve a classical planner's performance.

\section{References}

1. Alford R (2013) Search complexities for HTN planning. Ph.D. thesis, University of Maryland, College Park

2. Alford R, Bercher P, Aha DW (2015) Tight bounds for HTN planning. In: Proceedings of the 25th international conference on automated planning and scheduling (ICAPS). AAAI Press, pp 7-15

3. Alford R, Bercher P, Aha DW (2015) Tight bounds for HTN planning with task insertion. In: Proceedings of the 24th international joint conference on artificial intelligence (IJCAI). AAAI Press, pp 1502-1508

4. Alford R, Kuter U, Nau D, Goldman RP (2014) Plan aggregation for strong cyclic planning in nondeterministic domains. Artif Intell 216:206-232

5. Behnke G, Höller D, Biundo S (2015) On the complexity of HTN plan verification and its implications for plan recognition. In: Proceedings of the 25th international conference on automated planning and scheduling (ICAPS). AAAI Press, pp 25-33

6. Bercher P, Biundo S, Geier T, Hörnle T, Nothdurft F, Richter F, Schattenberg B (2014) Plan, repair, execute, explain-how planning helps to assemble your home theater. In: Proceedings of the 24th international conference on automated planning and scheduling (ICAPS). AAAI Press, pp 386-39

7. Breuer T, Macedo GRG, Hartanto R, Hochgeschwender N, Holz D, Hegger F, Jin Z, Müller C, Paulus J, Reckhaus M, Anotonio J, Ruizi Á, Plöger PG, Kraetzschmar GK (2012) Johnny: an autonomous service robot for domestic environments. J Intell Robot Syst 66(1-2):245-272
8. Erol K, Hendler J, Nau DS (1994) HTN planning: complexity and expressivity. In: Proceedings of the 12th national conference on artificial intelligence (AAAI), vol 94. AAAI Press, pp 1123-1128

9. Geier T, Bercher P (2011) On the decidability of HTN planning with task insertion. In: Proceedings of the 22nd international joint conference on artificial intelligence (IJCAI). AAAI Press, pp 1955-1961

10. Ghallab M, Nau DS, Traverso P (2004) Automated planning: theory \& practice. Morgan Kaufmann, Burlington, Massachusetts, USA

11. Helmert M (2006) The fast downward planning system. J Artif Intell Res 26(1):191-246

12. Hoffmann J, Nebel B (2001) The FF planning system: fast plan generation through heuristic search. J Artif Intell Res 14:253-302

13. Kuter U, Burstein M, Benton J, Thayer J, McCoy S (2015) HACKAR: helpful advice for code knowledge and attack resilience. In: Proceedings of the twenty-seventh IAAI conference. AAAI Press, pp 3987-3992

14. Nau DS, Au TC, Ilghami O, Kuter U, Wu D, Yaman F, MuñozAvila H, Murdock JW (2005) Applications of SHOP and SHOP2. Intell Syst IEEE 20:34-41

15. Richter S, Westphal M (2010) The LAMA planner: guiding costbased anytime planning with landmarks. J Artif Intell Res 39(1):127-177

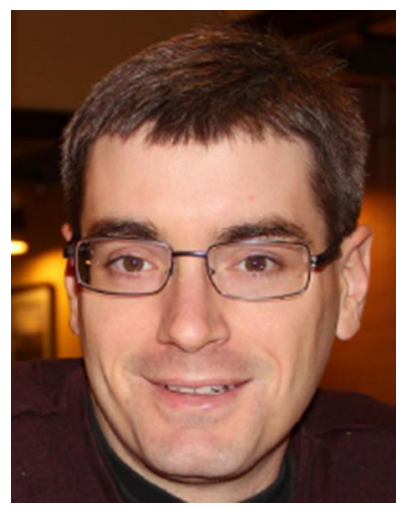

Ronald Alford graduated from the University of Maryland in 2014, and is now an ASEE postdoctoral researcher at the US Naval Research Laboratory. $\mathrm{He}$ specializes in automated planning with procedural knowledge. 